\title{
Resultados refracionais do retratamento de LASIK com ablação personalizada versus ablação convencional
}

\author{
Refractional results of LASIK retreatment with wavefront-guided ablation \\ versus standard ablation
}

\author{
Andréia Peltier Urbano ${ }^{1}$ \\ Walton Nosé ${ }^{2}$
}

Trabalho realizado no Departamento de Oftalmologia da Universidade Federal de São Paulo - UNIFESP - São Paulo (SP) - Brasil.

${ }^{1}$ Doutora em Medicina pelo Curso de Pós-Graduação em Oftalmologia e Ciências Visuais da Universidade Federal de São Paulo - UNIFESP - São Paulo (SP) - Brasil.

${ }^{2}$ Livre Docente do Departamento de oftalmologia da UNIFESP - São Paulo (SP) - Brasil. Professor Titular do Departamento de Oftalmologia. Universidade Metropolitana de Santos - UNIMES - Santos (SP) - Brasil.

Endereço para Correspondência: Andréia Peltier Urbano. Av. Cardeal da Silva, 2.070 - 201B - Salvador (BA) CEP 40231-250

E-mail:andreiaurbano@terra.com.br

Recebido para publicação em 09.05.2007

Última versão recebida em 14.06.2008

Aprovação em 22.06.2008

\begin{tabular}{|l|}
\hline RESUMO \\
\hline Objetivo: Avaliar os resultados do retratamento personalizado (Zyoptix, \\
Bausch \& Lomb) e do retratamento convencional (Planoscan, Bausch \& \\
Lomb) em olhos submetidos a LASIK primário convencional. Métodos: Foi \\
realizado estudo prospectivo, randomizado, de 74 olhos de 37 pacientes \\
submetidos aretratamento deLASIK para correção demiopia eastigmatismo. \\
Cada paciente foi submetido a retratamento de LASIK com ablação \\
personalizada em um olho e ablação convencional no olho contralateral. \\
Foram comparadas a acuidade visual de alto contraste e refração manifesta, \\
com acompanhamento de seis meses. Resultados: Os retratamentos per- \\
sonalizados de LASIK (Zyoptix) e os retratamentos convencionais de \\
LASIK (Planoscan) apresentaram valores estatisticamente similares para \\
a acuidade visual, ganho/perda de linhas da melhor acuidade visual \\
corrigida, índices de segurança e eficácia e porcentagem de olhos com \\
equivalente esférico(EE) entre $\pm 0,50$ D e 1,00 D no período pós-operatório \\
de seis meses. Houve diferença significativa para os valores do componen- \\
te esférico e do equivalente esférico nos períodos pós-operatórios de uma \\
semana, um, três e seis meses entre os grupos Zyoptix e Planoscan. Os \\
olhos do grupo Zyoptix apresentaram valores mais hipermétropes do que \\
os olhos do grupo Planoscan. A previsibilidade da correção do equivalen- \\
te esférico no grupo Zyoptix foi superior à do grupo Planoscan. Conclu- \\
sões: Aablação personalizada proporciona melhores resultados refracionais \\
do que a ablação convencional para os retratamentos de LASIK conven- \\
cional. A medida da acuidade visual de alto contraste não é parâmetro \\
sensível para evidenciar a melhor técnica cirúrgica.
\end{tabular}

Descritores: Erros de refração; Córnea; Ceratomileuse assistida por excimer laser in situ; Retratamento; Lasers

\section{INTRODUCÃO}

No panorama atual da cirurgia refrativa, a foto-ablação personalizada guiada pela análise de frentes de ondas tem sido objeto de infindas pesquisas no intuito de proporcionar uma correção refrativa com melhor qualidade visual pós-operatória.

A análise de frentes de ondas (wavefront), utilizada inicialmente na Astronomia, começou a ser utilizada na Oftalmologia com o objetivo de detecção das aberrações oculares. Desde 1962, Smirnov ${ }^{(1)}$ já havia referido que os olhos humanos apresentavam aberrações de alta ordem. Em 1997, Liang et al. (2) demonstraram que a correção clínica das aberrações oculares de alta ordem com o uso do Sistema Óptico Adaptativo proporcionava melhora da 
qualidade visual, com aumento da acuidade visual (visão "supernormal"). Em 1999, Theo Seiler e Marguerite McDonald, em centros distintos, realizaram as primeiras cirurgias personalizadas baseadas na análise de frentes de ondas, com o objetivo de corrigir as aberrações oculares de baixa e alta ordem em olhos humanos ${ }^{(3)}$.

A análise de frentes de ondas é uma nova modalidade diagnóstica e cirúrgica que permite a análise da refração ocular sob a perspectiva da Óptica Física - a óptica que estuda a luz como ondas. No processo fisiológico da refração ocular, os raios e frentes de onda procedentes de um objeto ao atravessarem o sistema óptico ocular sofre a refração pela córnea, cristalino e meios oculares e são focalizados em pontos distintos na retina, influenciados pelas aberrações oculares ${ }^{(4)}$. As aberrações oculares podem ser classificadas em aberrações de baixa ordem e em aberrações de alta ordem. Do ponto de vista prático, as aberrações de baixa ordem de segunda ordem - o defocus e o astigmatismo - equivalem aos componentes esfero-cilíndrico da refração clínica e podem ser corrigidas clinicamente através do uso de óculos, lentes de contato, ou cirurgicamente, pela técnica da cirurgia refrativa com a foto-ablação convencional baseada na fórmula de Munnerlyn ou com a foto-ablação personalizada baseada na análise de frentes de ondas. As aberrações de alta ordem, no entanto, podem ser corrigidas ou minimizadas cirurgicamente pela técnica da cirurgia refrativa personalizada baseada na análise de frentes de ondas $^{(5)}$.

Atualmente sabe-se que as aberrações oculares de alta ordem estão associadas à qualidade visual ${ }^{(6-7)}$. Olhos com grande quantidade de aberrações oculares de alta ordem apresentam alteração da qualidade visual, como pode ser observado especialmente nos casos de doenças oculares ou após cirurgias oculares. Está bem demonstrado que a cirurgia refrativa convencional tende a induzir aberrações oculares de alta ordem enquanto corrige as aberrações oculares de baixa ordem. Por este motivo, os pacientes podem apresentar queixas visuais pós-operatórias como ofuscamento, halos, sintomas de explosão de estrelas, visão dupla e outros distúrbios de visão noturna ${ }^{(7)}$. A cirurgia refrativa personalizada baseada na análise de frentes de ondas surgiu com o objetivo de corrigir ou minimizar as aberrações oculares de alta ordem enquanto corrige as aberrações de baixa ordem. Sendo assim, pode proporcionar melhor qualidade visual pós-operatória.

Os resultados das cirurgias refrativas personalizadas realizadas em olhos sem cirurgias prévias (cirurgias primárias) têm sido promissores quando comparados aos resultados das cirurgias refrativas convencionais ${ }^{(3,8)}$. Os resultados das cirurgias personalizadas nos retratamentos refrativos têm se mostrado ainda mais superiores do que as cirurgias personalizadas em olhos virgens de tratamento quanto à correção das aberrações oculares e quanto à melhora da qualidade visual em relação ao período pré-operatório ${ }^{(9)}$.

Com o objetivo de avaliar qual a melhor modalidade de ablação para os casos de retratamento refrativo, optamos por realizar um estudo comparativo dos resultados do retratamen- to de LASIK realizado com as técnicas de ablação personalizada e convencional.

\section{OBJETIVO}

Comparar os resultados cirúrgicos do retratamento com as técnicas de foto-ablação personalizada e foto-ablação convencional em olhos submetidos à cirurgia de LASIK primária convencional.

\section{MÉTODOS}

Foi realizado um estudo prospectivo, randomizado, de 74 olhos de 37 pacientes submetidos a retratamento de LASIK para correção de miopia e astigmatismo.

O grupo de estudo foi constituído por um dos olhos de cada paciente submetido a retratamento de LASIK com ablação personalizada Zyoptix (Bausch \& Lomb, Munique, Baviera). O grupo-controle foi composto pelo olho contralateral submetido a retratamento de LASIK com ablação convencional Planoscan (Bausch \& Lomb, Munique, Baviera). A escolha do olho a ser submetido ao retratamento com a técnica personalizada ou convencional foi aleatória.

Os pacientes realizaram cirurgia de LASIK primária com a técnica convencional, no período de novembro de 1995 a fevereiro de 2003, por um único cirurgião, na Eye Clinic Day Hospital (São Paulo, Brasil). Os retratamentos de LASIK foram realizados, no período de agosto de 2002 a outubro de 2003, por um único cirurgião, na Eye Clinic Day Hospital (São Paulo, Brasil). Após a cirurgia de retratamento de LASIK, os pacientes foram acompanhados por um período de seis meses.

Todos os pacientes assinaram consentimento, sendo devidamente informados, após explanação do procedimento, de potenciais benefícios e riscos. O estudo foi avaliado e aprovado pelo Comitê de Ética da Universidade Federal de São Paulo.

Os critérios de inclusão foram: cirurgia de LASIK primária prévia, bilateral e com correção pela técnica convencional; erros refrativos similares antes da cirurgia primária (equivalente esférico inferior a uma dioptria entre os dois olhos); erros refrativos residuais similares antes do retratamento (equivalente esférico inferior a uma dioptria entre os dois olhos) e idade superior a 18 anos.

Os critérios de exclusão foram: distúrbios oculares além de erros refrativos; doenças sistêmicas com manifestações oculares; cirurgias intra-oculares prévias (facectomia e vitrectomia); ocorrência de mais de uma cirurgia refrativa em cada olho; técnicas cirúrgicas diferentes entre os dois olhos; melhor acuidade visual corrigida inferior a 20/25; diferença de acuidade visual entre os dois olhos superior a uma linha da melhor acuidade visual corrigida e contra-indicação cirúrgica para nova cirurgia de LASIK. O retratamento de LASIK foi contra-indicado quando o valor calculado presumido para o estroma residual no pós-operatório era inferior a 250 micrômetros $(\mu \mathrm{m})$ e o valor calculado presumido para a ceratometria corneana pós-operató- 
ria inferior a 37 dioptrias (D). O retratamento de LASIK personalizado foi contra-indicado nos casos em que a aberrometria não era confiável.

No pré-operatório os pacientes foram submetidos a exame oftalmológico completo, incluindo medida da acuidade visual de alto contraste não-corrigida e corrigida, utilizando a tabela $E T D R S^{\mathrm{TM}}$ (Lighthouse Inc., Nova Iorque, Nova Iorque); refração clínica dinâmica e sob cicloplegia; paquimetria ultra-sônica com paquímetro Paradigm Ultrasound Pachymetric Analyzer, modelo P55 US (Paradigm Medical Inc., South Lake, Utah); topografia tridimensional da córnea com o topógrafo Orbscan II Z TMOrbtek / Bausch \& Lomb, South Lake City, Utah); aberrometria com sensor de frente de ondas Zywave ${ }^{\text {TY }}$ tipo ShackHartmann) - versão 3.21 (Bausch \& Lomb, Munique, Baviera).

Todas as cirurgias de retratamento foram realizadas por um único cirurgião, pela técnica cirúrgica de LASIK personalizado Zyoptix (grupo de estudo) e pela técnica cirúrgica de LASIK convencional Planoscan (grupo-controle). Foi procedido o levantamento do disco em todos os pacientes e ablação do estroma corneano foi realizada com o aparelho de excimer laser Technolas 217Z 7 Bausch \& Lomb, Munique, Baviera). O retratamento convencional com a plataforma Planoscan foi realizado de acordo com o nomograma convencional para cirurgias primárias, baseado na Lei de Munnerlyn. A correção dos componentes esférico, cilíndrico e eixo da refração ocular foi realizada com aplicação de um feixe de laser de $2 \mathrm{~mm}$. O retratamento personalizado com a plataforma Zyoptix foi realizado de acordo com o planejamento do tratamento realizado no Programa Zylink versão 2.3 (Bausch \& Lomb, Munique, Baviera). A refração utilizada para o retratamento foi baseada no menor valor esférico e cilíndrico da refração ocular comparando-se os valores da refração dinâmica, refração estática e refração da aberrometria. O programa de tratamento personalizado Zylink permite a modificação dos componentes esférico e cilíndrico da correção desejada, sem, no entanto, permitir a modificação do eixo do cilindro - que tem que ser do exame da aberrometria. Desta maneira, foi escolhido o exame de aberrometria que apresentava o eixo mais próximo da refração clínica do paciente. Um feixe de laser inicial de $2 \mathrm{~mm}$ foi utilizado para correção dos componentes esférico e cilíndrico, com posterior diminuição deste feixe para $1 \mathrm{~mm}$, para correção das aberrações oculares de alta ordem.

Para o período pós-operatório, foram prescritos para todos os pacientes colírios de: tobramicina a $0,3 \%$, cetorolaco de trometamina a $0,5 \%$, acetato de prednisolona a $0,12 \%$ e hipromelose a $0,5 \%$.

Os pacientes foram examinados nos períodos pós-operatórios de 1 dia, 1 semana, um, três e seis meses após a cirurgia. Toda a avaliação pré e pós-operatória foi realizada por um mesmo examinador. Os resultados da acuidade visual foram descritos com a análise dos valores obtidos no sexto mês pósoperatório e os resultados dos erros refrativos foram descritos com a análise dos valores obtidos no primeiro dia, primeira semana, primeiro, terceiro e sexto meses pós-operatórios. Foi procedida a aberrometria pós-operatória nos pós-operatórios de um mês, três meses e seis meses. A comparação dos resulta- dos das aberrações oculares será abordada em publicação posterior.

Considerou-se como índice de segurança de 1,00 a manutenção da melhor acuidade visual de alto contraste corrigida e índice de eficácia de 1,00 uma acuidade visual de alto contraste não-corrigida no pós-operatório igual à melhor acuidade visual de alto contraste corrigida no pré-operatório.

\section{Método estatístico}

A análise estatística foi realizada com o Programa Statistical Package for the Social Sciences SPSS (SPSS Inc., Chicago, Illinois) na versão 10.0 para Windows. As variáveis contínuas foram descritas pela média e desvio-padrão e as variáveis categóricas foram descritas em valores porcentuais. $\mathrm{O}$ valor da significância estatística (valor do $p$ ) foi obtido através de testes não-paramétricos de Mann-Whitney (para a comparação inter-grupo) e de Wilcoxon (para a comparação intragrupo). $\mathrm{O}$ valor do $p$ foi considerado estatisticamente significante quando $<0,05$.

\section{RESULTADOS}

A idade média dos 37 pacientes foi 32,24 $\pm 07,02$ (DP) anos, variando de 20 a 52 anos. Vinte e três pacientes eram do sexo feminino e 14 do sexo masculino.

A média do equivalente esférico antes da cirurgia refrativa era de $-5,37 \pm 2,68 \mathrm{D}$, variando de $-13,00 \mathrm{a}+0,88 \mathrm{D}$. A média de tempo entre a cirurgia primária e o retratamento foi de $18,07 \pm 16,26$ meses, com período mínimo de três meses e período máximo de 84 meses.

No período pré-operatório do retratamento de LASIK, os exames realizados para avaliação da indicação cirúrgica evidenciaram que os olhos dos grupos Zyoptix e Planoscan foram similares quanto aos valores da acuidade visual de alto contraste não-corrigida e corrigida; do EE da refração clínica e da refração da aberrometria com a pupila não-dilatada e dilatada; do tamanho da pupila não-dilatada e dilatada e das aberrações oculares $(\mathrm{p}>0,05)$.

A análise comparativa dos valores intra-operatórios entre os grupos Zyoptix e Planoscan está apresentada na tabela 1. Os valores do equivalente esférico do tratamento e da zona óptica foram similares entre os dois grupos $(p>0,05)$. A profundidade de ablação e a quantidade de pulsos aplicados foram estatisticamente superiores no grupo Zyoptix ( $\mathrm{p}=0,01$ ).

Não houve complicações intra-operatórias ou pós-operatórias, como crescimento epitelial na interface, ceratite lamelar difusa ou infecções, nos retratamentos de LASIK com as técnicas Zyoptix e Planoscan.

Durante o período do acompanhamento do estudo, todos os 37 pacientes $(100 \%)$ compareceram à consulta oftalmológica no primeiro mês, $29(78,3 \%)$ compareceram no terceiro mês e $30(81 \%)$ retornaram no sexto mês.

No período pré-operatório do retratamento, a acuidade visual corrigida ( $\mathrm{AV} \mathrm{c} / \mathrm{c}$ ) foi $\geq 20 / 25 \mathrm{em}$ todos os casos. No 
grupo Zyoptix, 94,4\% dos olhos apresentaram AV c/c $\geq 20 / 20$ e $22,2 \% \geq 20 / 16$. No grupo Planoscan, $88,9 \%$ apresentaram AV $\mathrm{c} / \mathrm{c} \geq 20 / 20$ e $22,2 \% \geq 20 / 16(\mathrm{p}=0,69)$.

No pós-operatório de seis meses, no grupo Zyoptix, 93,3\% dos olhos apresentaram acuidade visual sem correção (AV s/c) $\geq 20 / 20,63,3 \% \geq 20 / 16$ e $23,3 \% \geq 20 / 12,5$. No grupo Planoscan, $86,7 \%$ apresentaram AV s/c $\geq 20 / 20,50 \% \geq 20 / 16$ e $6,7 \% \geq 20 / 12,5$, sem diferença estatística entre os grupos $(\mathrm{p}=0,11)$ (Figura 1).

Em relação a acuidade visual corrigida no sexto mês pósoperatório, no grupo Zyoptix, 93,4\% apresentaram AV c/c $\geq 20 / 20$, $63,4 \% \geq 20 / 16$ e $26,7 \% \geq 20 / 12,5$. No grupo Planoscan, $91 \%$ apresentaram AV c/c $\geq 20 / 20,67,7 \% \geq 20 / 16$ e $6,7 \% \geq 20 / 12,5$, sem diferença estatística entre os grupos $(\mathrm{p}=0,40)$ (Figura 2).

Em relação à perda/ganho de linhas da melhor acuidade visual corrigida, no acompanhamento de seis meses, houve perda de uma linha da melhor acuidade visual corrigida em 3,3\% dos olhos no grupo Zyoptix e em 13,3\% dos olhos no grupo Planoscan. No grupo Zyoptix, 33,3\% ganharam uma linha e $13,3 \%$ ganharam duas linhas, enquanto no grupo Planoscan $50 \%$ ganharam uma linha, sem diferença significativa entre os grupos $(p=0,06)$ (Figura 3$)$.

No sexto mês pós-operatório, o índice de segurança foi $1,16 \pm$ 0,22 para o grupo Zyoptix e 1,10 $\pm 0,17$ para o grupo Planoscan $(\mathrm{p}=0,35)$ e o índice de eficácia foi $1,15 \pm 0,22$ para o grupo Zyoptix e $1,06 \pm 0,20$ para o grupo Planoscan $(p=0,20)$.

Os valores das refrações manifestas do período pré-operatório do LASIK primário e do retratamento de LASIK estão apresentados na tabela $2 \mathrm{~A}$, enquanto os valores das refrações manifestas do período pós-operatório do retratamento de LASIK estão nas tabelas 2 B e 2 C.

Os grupos Zyoptix e Planoscan apresentaram valores similares dos componentes esférico, cilíndrico e de equivalente esférico antes do LASIK primário e antes do retratamento de LASIK.

No acompanhamento pós-operatório de uma semana, um mês, três meses e seis meses após o retratamento, houve diferença estatística dos valores dos componentes esféricos e dos equivalentes esféricos entre os grupos Zyoptix e Planoscan. Os olhos do grupo Zyoptix ficaram com médias mais próximas da emetropia, com tendência hipermétrope, enquanto que os olhos

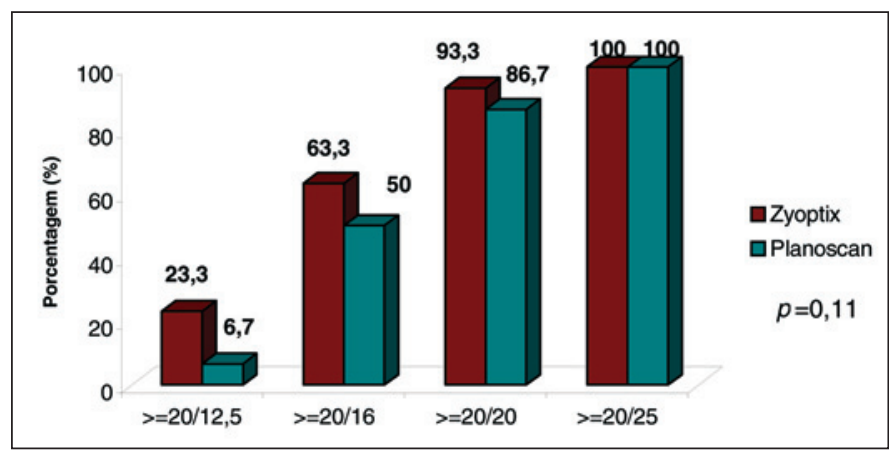

Figura 1 - Comparação da acuidade visual de alto contraste nãocorrigida entre os grupos Zyoptix e Planoscan em seis meses

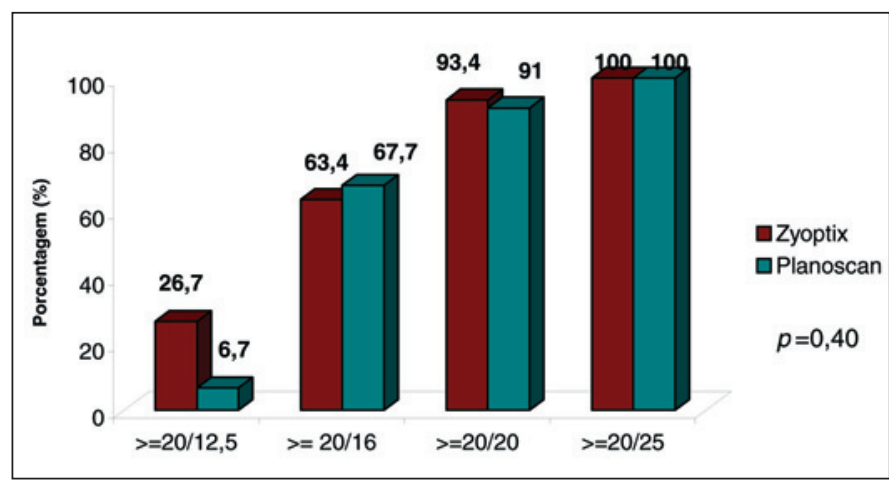

Figura 2 - Comparação da acuidade visual de alto contraste corrigida entre os grupos Zyoptix e Planoscan em seis meses

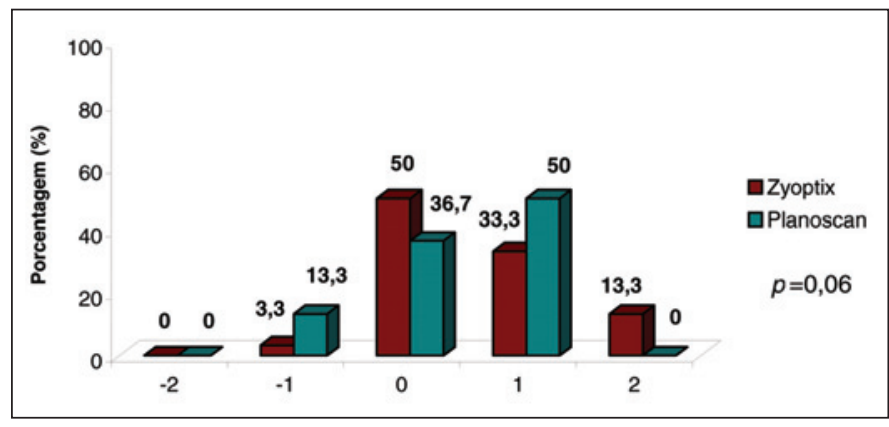

Figura 3 - Comparação entre perda/ganho de linhas da acuidade visual de alto contraste corrigida nos grupos Zyoptix e Planoscan em seis meses

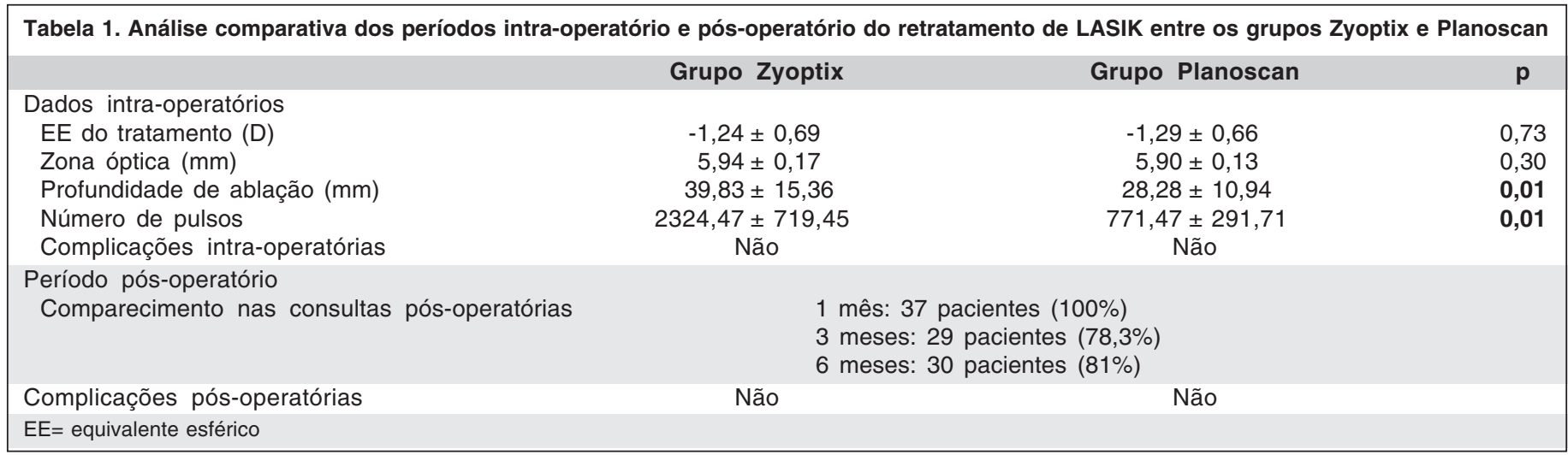




\begin{tabular}{|c|c|c|c|c|c|c|}
\hline & \multicolumn{6}{|c|}{ Refração } \\
\hline & \multicolumn{3}{|c|}{ Antes do LASIK primário } & \multicolumn{3}{|c|}{ Antes do retratamento do LASIK } \\
\hline & Zyoptix & Planoscan & $p$ & Zyoptix & Planoscan & $p$ \\
\hline Esf (D) & $-4,81 \pm 2,98$ & $-4,73 \pm 2,67$ & 0,97 & $-1,07 \pm 0,81$ & $-1,07 \pm 0,73$ & 0,77 \\
\hline Cil (D) & $-1,22 \pm 1,11$ & $-1,17 \pm 1,16$ & 0,77 & $-0,53 \pm 0,45$ & $-0,56 \pm 0,44$ & 0,65 \\
\hline EE (D) & $-5,42 \pm 2,82$ & $-5,32 \pm 2,56$ & 0,96 & $-1,34 \pm 0,81$ & $-1,33 \pm 0,74$ & 0,91 \\
\hline
\end{tabular}

\begin{tabular}{|c|c|c|c|c|c|c|}
\hline & \multicolumn{6}{|c|}{ Refração } \\
\hline & \multicolumn{3}{|c|}{ Um dia } & \multicolumn{3}{|c|}{ Uma semana } \\
\hline & Zyoptix & Planoscan & $p$ & Zyoptix & Planoscan & $p$ \\
\hline Esf (D) & $+0,12 \pm 0,36$ & $-0,02 \pm 0,22$ & 0,13 & $+0,13 \pm 0,38$ & $-0,09 \pm 0,31$ & 0,02 \\
\hline Cil (D) & $-0,15 \pm 0,39$ & $-0,11 \pm 0,23$ & 0,98 & $-0,15 \pm 0,22$ & $-0,20 \pm 0,23$ & 0,28 \\
\hline EE (D) & $+0,04 \pm 0,43$ & $-0,10 \pm 0,27$ & 0,13 & $+0,02 \pm 0,40$ & $-0,21 \pm 0,33$ & 0,01 \\
\hline
\end{tabular}

\begin{tabular}{|c|c|c|c|c|c|c|c|c|c|}
\hline \multicolumn{10}{|c|}{$\begin{array}{c}\text { Tabela } 2 \text { C. Refração manifesta pós-operatória nos grupos Zyoptix e Planoscan em um mês, três meses e seis meses } \\
\text { Refração }\end{array}$} \\
\hline & \multicolumn{3}{|c|}{ Um mês } & \multicolumn{3}{|c|}{ Três meses } & \multicolumn{3}{|c|}{ Seis meses } \\
\hline & Zyoptix & Planoscan & $p$ & Zyoptix & Planoscan & $p$ & Zyoptix & Planoscan & $p$ \\
\hline Esf (D) & $+0,19 \pm 0,30$ & $-0,06 \pm 0,29$ & 0,01 & $+0,07 \pm 0,28$ & $-0,12 \pm 0,22$ & 0,01 & $+0,14 \pm 0,37$ & $-0,10 \pm 0,22$ & 0,01 \\
\hline Cil (D) & $-0,12 \pm 0,18$ & $-0,21 \pm 0,21$ & 0,05 & $-0,16 \pm 0,22$ & $-0,18 \pm 0,22$ & 0,61 & $-0,13 \pm 0,18$ & $-0,18 \pm 0,18$ & 0,29 \\
\hline EE (D) & $-0,12 \pm 0,32$ & $-0,18 \pm 0,31$ & 0,13 & $-0,03 \pm 0,31$ & $-0,22 \pm 0,27$ & 0,02 & $+0,02 \pm 0,32$ & $-0,20 \pm 0,25$ & 0,01 \\
\hline
\end{tabular}

do grupo Planoscan ficaram com tendência míope após o retratamento. Não houve diferença dos valores dos componentes cilíndricos entre os dois grupos.

Os resultados da análise do efeito da regressão do EE após o retratamento, realizada, separadamente, para os grupos Zyoptix e Planoscan, estão apresentados, respectivamente, nas figuras 4 e 5 .

No grupo Zyoptix, a diferença dos valores do EE entre os períodos de um mês e três meses e a diferença entre os períodos de um mês e seis meses foi estatisticamente significante. Houve uma variação do $\mathrm{EE}$ de $-0,18 \pm 0,32 \mathrm{D}$ entre o primeiro e o terceiro meses $(p=0,01)$ e esta variação foi de $-0,13 \pm 0,31 \mathrm{D}$ entre $o$ primeiro e o sexto meses $(p=0,04)$. Não houve diferença dos valores entre os demais períodos analisados. Quando foram verificadas as diferenças dos valores dos componentes esféricos e do componente cilíndrico entre os períodos pós-operatórios, foi observado que a regressão do EE obtida entre o primeiro e o terceiro e entre o primeiro e o sexto meses ocorreu em razão do componente esférico. $\mathrm{O}$ valor do componente cilíndrico não modificou durante o período pós-operatório.

No grupo Planoscan, não houve diferença estatística para os valores do EE do componente esférico e do componente cilíndrico durante o período do acompanhamento.

No acompanhamento de seis meses, o equivalente esférico estava entre $\pm 0,50 \mathrm{D}$ da emetropia em 93,3\% do grupo Zyoptix e em $86,7 \%$ do grupo Planoscan $(\mathrm{p}=0,39)$ e todos os olhos estavam entre $\pm 1,00 \mathrm{D}$ (Figura 6).

A previsibilidade da correção do equivalente esférico, que foi estudada através da correlação entre a correção refrativa desejada (EE desejado) e a obtida (EE obtido), está apresentada como gráfico de dispersão. Para o pós-operatório de seis meses, o coeficiente de correlação ajustado $\left(\mathrm{R}^{2}\right)$ foi 0,84 para o grupo Zyoptix ( $\mathrm{p}=0,01)$ e 0,70 para o grupo Planoscan $(\mathrm{p}=0,01)$ (Figura 7).

\section{DISCUSS ÃO}

A cirurgia personalizada baseada na análise de frente de onda pode ter muitas vantagens sobre as técnicas tradicionais ${ }^{(10)}$. Os estudos preliminares dos resultados das cirurgias personalizadas em olhos virgens de tratamento refrativo evidenciaram resultados refrativos satisfatórios. Os estudos comparativos entre as técnicas de ablação personalizada e convencional para cirurgias refrativas primárias têm apresentado bons resultados com as duas técnicas avaliadas. Neste estudo avaliamos qual das técnicas seria a melhor para o retratamento de LASIK primário convencional.

Podemos observar nos resultados apresentados que o retratamento personalizado Zyoptix e o retratamento convencional 


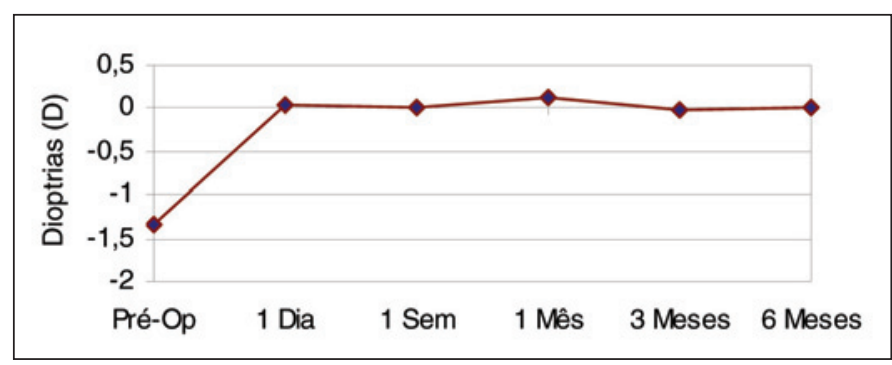

Figura 4 - Mudança do equivalente esférico no grupo Zyoptix

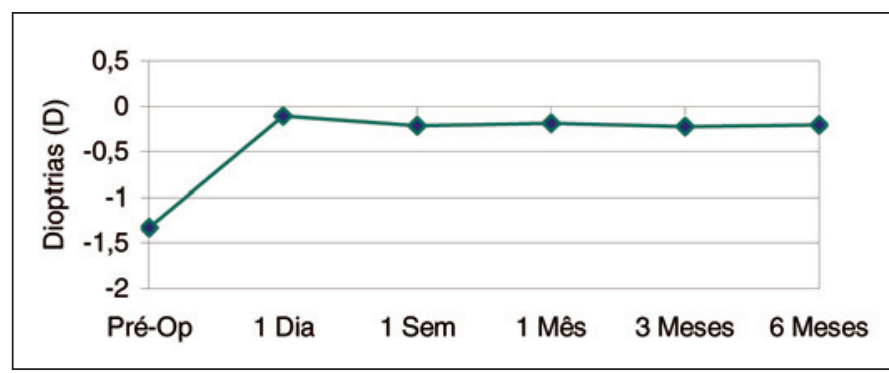

Figura 5 - Mudança do equivalente esférico no grupo Planoscan

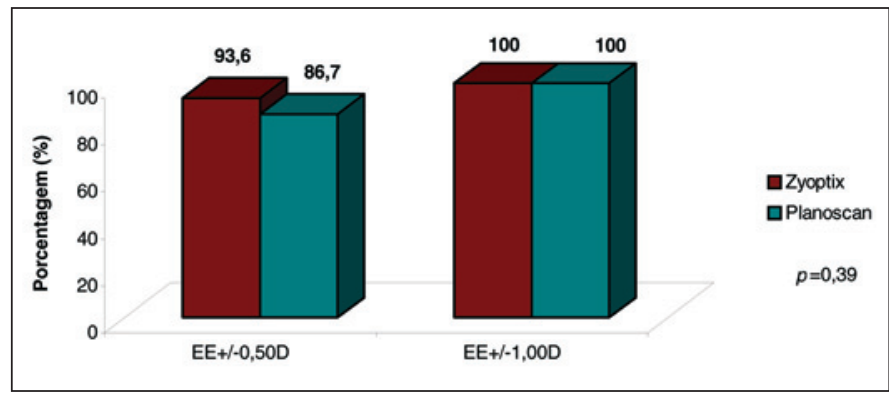

Figura 6 - Equivalente esférico obtido nos grupos Zyoptix e Planoscan no sexto mês

Planoscan foram estatisticamente equivalentes quanto à acuidade visual de alto contraste corrigida e não-corrigida, perda/ ganho de linhas da melhor acuidade visual corrigida, segurança e eficácia e porcentagem de olhos próximos à emetropia.

Os estudos comparativos dos resultados da ablação personalizada e convencional para cirurgia de LASIK primária também não encontraram diferença estatística em relação à acuidade visual de alto contraste não-corrigida ${ }^{(11-15)}$. Este resultado já poderia ser esperado, pois já está bem demonstrado que a acuidade visual de alto contrate não é um parâmetro sensível de qualidade visual ${ }^{(16)}$.

Nos trabalhos publicados sobre cirurgia personalizada para LASIK primário, a porcentagem de acuidade visual nãocorrigida superior a $20 / 20$ varia de $50 \%{ }^{(17)}$ a $98 \%{ }^{(18)}$ e a da acuidade visual não-corrigida superior a $20 / 15$ varia entre $0^{(19)}$ e $80 \%{ }^{(17)}$. Nos trabalhos publicados sobre cirurgia personalizada para retratamento refrativo, a porcentagem de acuidade visual não-corrigida superior a $20 / 20$ varia de $25 \%^{(20)}$ a $85 \%^{(21)}$.
Na literatura, os valores para a acuidade visual corrigida igual ou superior a 20/20 nos estudos sobre LASIK primário personalizado variam de $56 \%{ }^{(19)}$ a $100 \%{ }^{(22)}$, Nos estudos sobre retratamento personalizado variam de $84,4 \%{ }^{(9)}$ a $100 \%^{(21)}$ e nos estudos sobre o retratamento convencional de LASIK de até $92,9 \%{ }^{(23)}$.

Os demais estudos que compararam as técnicas de LASIK primário convencional e personalizado também não verificaram diferença de perda ou ganho de linhas da melhor acuidade visual corrigida, entre as duas técnicas cirúrgicas ${ }^{(11-12,15)}$. Como podemos observar, no pós-operatório de cirurgias refrativas pode haver ganho de linhas da melhor acuidade visual corrigida. Segundo Applegate e Howland ${ }^{(24)}$, a melhora da acuidade visual corrigida após a cirurgia refrativa resulta do aumento do tamanho da imagem retiniana proporcionado pela correção do erro refrativo miópico no plano corneano.

Quando analisamos os índices de segurança de um estudo, estamos verificando a capacidade do tratamento de manter ou não, no pós-operatório, a melhor acuidade visual corrigida que o paciente apresentava no pré-operatório e, por sua vez, ao analisarmos a eficácia, mensuramos se a acuidade visual não-corrigida no pós-operatório manteve-se ou não igual à melhor acuidade visual corrigida do pré-operatório. Em nosso estudo, estes índices foram superiores a 1,00 , indicando que a maioria da amostra teve melhora da acuidade visual no pós-operatório, apontando para o benefício do retratamento para estes pacientes.

A porcentagem de olhos com $\mathrm{EE} \pm 0,50 \mathrm{D}$ nos estudos sobre cirurgia personalizada primária varia de $67,7 \%$ a $100 \%{ }^{(19,25-26)}$. Nos estudos sobre retratamento personalizado, a faixa de equivalente esférico $\pm 0,50 \mathrm{D}$ encontrada é, em torno, de $90 \%^{(21,27)}$. Nos estudos sobre retratamento de LASIK convencional, estas porcentagens são muito variáveis ${ }^{(23,28-30)}$. Os resultados com baixa porcentagem de $\mathrm{EE} \pm 0,50 \mathrm{D}$ podem estar relacionados à amostra pequena ou à faixa da refração corrigida. Os estudos que descreveram resultados de $\mathrm{EE} \pm 0,50 \mathrm{D}$ acima de $70 \%$ apresentavam uma amostra com maior número de casos ou com faixa menor de equivalente esférico pré-operatório(23,28,30).

As principais diferenças encontradas entre o retratamento personalizado Zyoptix e o retratamento convencional Planoscan neste estudo foram relacionadas à correção do erro refrativo. Observamos que os grupos Zyoptix e Planoscan apresentavam componentes esférico, componente cilíndrico e equivalente esférico similares tanto antes do LASIK primário quanto antes do retratamento de LASIK. No primeiro dia após o retratamento, as refrações manifestas entre os dois grupos estavam semelhantes. A partir da primeira semana pós-operatória, começou a haver diferença estatística entre os valores refracionais dos dois grupos e esta tendência manteve-se até o último período avaliado de seis meses. A diferença encontrada para os valores do equivalente esférico foi devido às diferenças dos componentes esféricos, pois os valores dos componentes cilíndricos foram similares entre os dois grupos. Enquanto os componentes esféricos e equivalentes esféricos no grupo Zyoptix tinham médias mais hipermétropes, no grupo Planoscan, estes valores eram mais míopes. 


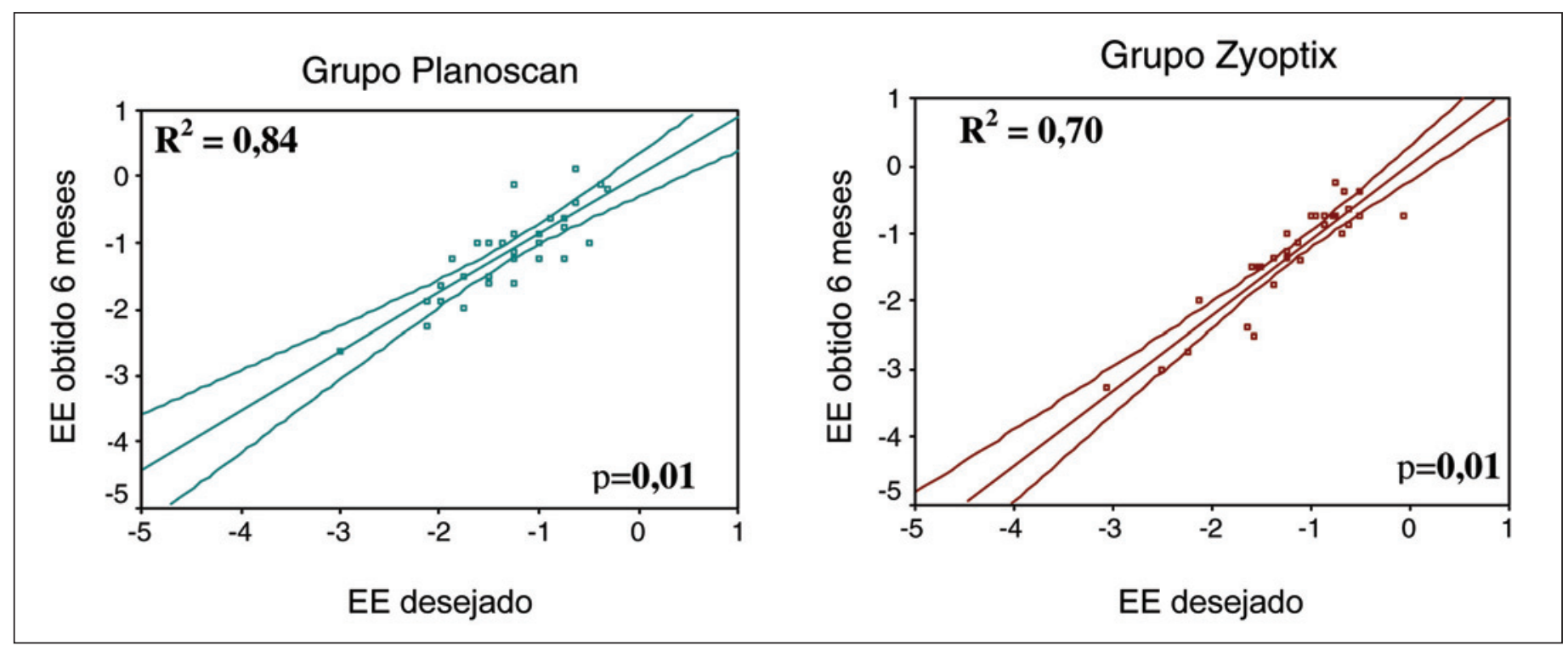

Figura 7 - Previsibilidade nos grupos Zyoptix e Planoscan. Correlação entre o equivalente esférico desejado (EE desejado) e o equivalente esférico obtido (EE obtido) em seis meses.

É importante observar que os resultados refrativos relacionados à refração manifesta no período pós-operatório nos tratamentos personalizados estão diretamente relacionados ao nomograma utilizado para o tratamento. Carones e et al. enfatizaram que o nomograma do tratamento é um dos aspectos mais importantes na determinação dos resultados da cirurgia personalizada e que especial atenção deve ser dedicada na acurácia do algoritmo de tratamento para correção do defocus para evitar hipercorreção ${ }^{(31)}$. Alguns cirurgiões preferem sistematicamente hipocorrigir o componente esférico ${ }^{(32)}$. Aizawa et al. tiveram que revisar o nomograma pois alguns casos de alta miopia resultaram em hipocorreção. Eles observaram que a utilização dos valores da refração manifesta melhoraram a previsibilidade do estudo ${ }^{(33)}$. Atenção especial no ajuste do nomograma deve ser utilizado para os casos de retratamento. Resultados preliminares de estudos com pequena amostra evidenciaram que o retratamento personalizado foi efetivo para retratamento de LASIK e PRK, porém com tendência à hipercorreção. Carones et al. ${ }^{(31)}$ observaram um equivalente esférico hipermetrópico em todos os sete olhos reportados, variando de $+0,12$ a $+1,50$ D. Acreditamos que o fato de termos utilizado um nomograma baseado na escolha do menor valor esférico e cilíndrico comparando-se os valores da refração dinâmica, estática e da refração da aberrometria tenha permitido melhor previsibilidade cirúrgica do que no retratamento convencional.

Quando analisamos a modificação do equivalente esférico durante o período do pós-operatório em nosso estudo, pudemos observar que no grupo Zyoptix houve uma redução significativa do valor da hipermetropia residual do primeiro para o sexto mês pós-operatório. Acreditamos que este processo possa estar relacionado ao fenômeno da acomodação, uma vez que a refração utilizada como referência para o acompanhamento foi a refração subjetiva manifesta. Esta tendência de modificação do equiva- lente esférico durante o pós-operatório no grupo Zyoptix do nosso estudo também foi observada por Aizawa et al. ${ }^{(33)}$. Na avaliação dos resultados das cirurgias de LASIK primárias personalizadas com a plataforma Zyoptix (Bausch \& Lomb, Munique, Baviera) realizada por estes autores, foi encontrada uma diferença estatística entre o equivalente esférico de $+0,11 \mathrm{D}$ na primeira semana para $-0,24 \mathrm{D}$ no primeiro mês e $-0,45 \mathrm{D}$ no terceiro mês. Após este período, houve estabilidade da refração que, no sexto mês, apresentou equivalente esférico de $-0,38$ D. É interessante observar que, de forma similar ao nosso estudo, os valores mais míopes foram encontrados no terceiro mês após a cirurgia. No estudo de Nuijts et al. ${ }^{(11)}$, os autores encontraram estabilidade refracional similar entre o grupo Zyoptix e Planoscan (Bausch \& Lomb, Munique, Baviera), em relação ao equivalente esférico de uma semana a seis meses. Apesar dos autores não terem descrito os valores exatos dos equivalentes esféricos nos diversos períodos, a análise do gráfico apresentado no estudo permite observar que os valores mais negativos ocorreram também no terceiro mês pós-operatório. Imaginamos que o terceiro mês pós-operatório seja o período crítico no processo da cicatrização pós-operatória ou período em que o fenômeno da acomodação ocularé mais pronunciado.

Observamos que no grupo Planoscan do nosso estudo a estabilidade refracional foi alcançada precocemente no pósoperatório, sem regressão do efeito terapêutico. Não foi encontrada diferença estatística na mudança do componente esférico, cilíndrico ou do equivalente esférico durante todo o período pós-operatório. Febbraro et al. ${ }^{(28)}$ também não encontraram diferença estatística entre os equivalentes esféricos no pós-operatório de um, três, seis e 12 meses do retratamento de LASIK convencional, apesar dos valores discretamente mais míopes no primeiro ano $(\mathrm{EE}=-0,13 \pm 0,33 \mathrm{D})$ em comparação ao do primeiro mês $(E E=+0,03 \pm 0,44 \mathrm{D})$. 


\section{CONCLUSÕES}

O presente estudo evidenciou que o retratamento personalizado Zyoptix foi superior do que o retratamento convencional Planoscan na correção do componente esférico refracional e do equivalente esférico da refração manifesta, com melhor previsibilidade cirúrgica e menor efeito de regressão. As medidas de acuidade visual no pós-operatório foram, no entanto, similares para as duas técnicas, sugerindo que os parâmetros de medida de acuidade visual de alto contraste não são os mais sensíveis para evidenciar a melhor técnica cirúrgica.

\section{AGRADECIMENTOS}

Agradecimento à Direção, colegas e funcionários da Eye Clinic pelo grande apoio e importante colaboração neste estudo.

\section{ABSTRACT}

Purpose: To evaluate refractive outcomes of wavefront-guided LASIK (Zyoptix, Bausch \& Lomb) versus standard LASIK (PlanoScan, Bausch \& Lomb) in retreatment of primary LASIK for myopia and myopic astigmatism. Methods: A prospective, randomized trial with paired eye control of 74 eyes from 37 patients who underwent LASIK retreatment was performed. Each patient underwent retreatment using Zyoptix LASIK in 1 eye and Planoscan LASIK in the contralateral eye. A complete ophthalmologic examination was performed, including evaluation of high contrast visual acuity and manifest refraction, with a follow-up of 6 months. Results: Both Zyoptix and Planoscan groups had similar values of high contrast visual acuity, gain and loss of best spectacle-corrected visual acuity, safety and efficacy indexes, percentage of eyes with spherical equivalent within $\pm 0,50 \mathrm{D}$ and $\pm 1,00 \mathrm{D}$ in the postoperative period of 6 months. In the Zyoptix group, the values of spherical component and spherical equivalent of manifest refraction were more hyperopic than in the Planoscan group in the postoperative periods of 1 week, 1, 3 and 6 months. The predictability of spherical equivalent correction was better for the Zyoptix group than for the Planoscan group at 6 months. Conclusions: Wavefront-guided LASIK with Zyoptix produces superior refractive outcomes than standard LASIK with Planoscan in retreatment of refractive errors after primary LASIK. High contrast visual acuity measures are not sensitive in showing the best technique.

Keywords: Refractive errors; Cornea; Keratomileusis, laser in situ; Retreatment; Lasers

\section{REFERÊNCIAS}

1. Smirnov MS. Measurement of the wave aberration in the human eye. Biopizika. 1961;6:776-95.
2. Liang J, Williams DR. Aberrations and retinal image quality of the normal human eye. J Opt Soc Am A Opt Image Sci Vis. 1997;14(11):2873-83.

3. Chalita MR, Chamon W, Schor P, Freda R, Krueger R. Resultados de literatura em tratamentos personalizados com excimer laser. In: Alves MR, Chamon W, Nosé W, editores. Cirurgia Refrativa. Rio de Janeiro: Cultura Médica; 2003. p.353-9.

4. Urbano AP, Leal M, Urbano I. Princípios básicos de óptica. In: Urbano AP, editor. Wavefront - Cirurgia Personalizada. Rio de Janeiro: Cultura Médica; 2006. p.39-55.

5. Urbano AP, Leal M, Urbano I. Descrição das aberrações oculares. In: Urbano AP, editor. Wavefront - Cirurgia personalizada. Rio de Janeiro: Cultura Médica; 2006. p.101-13.

6. Chalita MR, Chavala S, Xu M, Krueger RR. Wavefront analysis in post-LASIK eyes and its correlation with visual symptoms, refraction, and topography. Ophthalmology. 2004;111(3):447-53. Comment in: Ophthalmology. 2005;112(6):1171; author reply 1171 .

7. Chalita MR, Krueger RR. Correlation of aberrations with visual acuity and symptoms. Ophthalmol Clin North Am. 2004;17(2):135-42, v-vi.

8. Urbano AP. Cirurgias primárias personalizadas. In: Urbano AP, editor. Wavefront - Cirurgia Personalizada. Rio de Janeiro: Cultura Médica; 2006. p.375-95.

9. Urbano AP, Nosé R, Nosé W. Reoperações personalizadas. In: Alves MR, Chamon W, Nosé W, editores. Cirurgia refrativa. Rio de Janeiro: Cultura Médica; 2003. p.359-67.

10. Lyle WA, Jin GJ. Retreatment after initial laser in situ keratomileusis. J Cataract Refract Surg. 2000;26(5):650-9. Comment in: J Cataract Refract Surg. 2000;26(5):625-6.

11. Nuijts RM, Nabar VA, Hament WJ, Eggink FA. Wavefront-guided versus standard laser in situ keratomileusis to correct low to moderate myopia. J Cataract Refract Surg. 2002;28(11):1907-13.

12. Nagy ZZ, Palágyi-Deák I, Kelemen E, Kovács A. Wavefront-guided photorefractive keratectomy for myopia and myopic astigmatism. J Refract Surg. 2002; 18(5):S615-9.

13. Kim TI, Yang SJ, Tchah H. Bilateral comparison of wavefront-guided versus conventional laser in situ keratomileusis with Bausch and Lomb Zyoptix. J Refract Surg. 2004; 20(5):432-8.

14. Reinstein DZ, Neal DR, Vogelsang H, Schroeder E, Nagy ZZ, Bergt M, et al. Optimized and wavefront guided corneal refractive surgery using the Carl Zeiss Meditec platform: the WASCA aberrometer, CRS-Master, and MEL80 excimer laser. Ophthalmol Clin North Am. 2004; 17(2):191-210, vii.

15. Vongthongsri A, Phusitphoykai N, Naripthapan P. Comparison of wavefront-guided customized ablation vs. conventional ablation in laser in situ keratomileusis. J Refract Surg. 2002;18(3 Suppl):S332-5.

16. Pesudovs K, Marsack JD, Donnelly WJ $3^{\text {rd }}$, Thibos LN, Applegate RA. Measuring visual acuity-mesopic or photopic conditions, and high or low contrast letters? J Refract Surg. 2004;20(5):S508-14.

17. Durrie DS, Stahl J. Randomized comparison of custom laser in situ keratomileusis with the Alcon CustomCornea and the Bausch \& Lomb Zyoptix systems: one-month results. J Refract Surg. 2004;20(5):S614-8.

18. Awwad ST, El-Kateb M, Bowman RW, Cavanagh HD, McCulley JP. Wavefront-guided laser in situ keratomileusis with the Alcon CustomCornea and the VISX CustomVue: three-month results. J Refract Surg. 2004;20(5):S606-13.

19. Slade S. Contralateral comparison of Alcon CustomCornea and VISX CustomVue wavefront-guided laser in situ keratomileusis: one-month results. J Refract Surg. 2004;20(5):S601-5.

20. Castanera J, Serra A, Rios C. Wavefront-guided ablation with Bausch and Lomb Zyoptix for retreatments after laser in situ keratomileusis for myopia. J Refract Surg. 2004;20(5):439-43.

21. Winkler von Mohrenfels C, Huber A, Gabler B, Herrmann W, Kempe A, Donitzky C, Lohmann CP. Wavefront-guided laser epithelial keratomileusis with the wavelight concept system 500. J Refract Surg. 2004;20(5):S565-9.

22. Pettit GH, Campin JA, MacDonald MB, Krueger RR. Customized ablation using the Alcon CustomCornea platform. In: Krueger RR, Applegate RA, MacRae SM, editors. Wavefront customized visual correction: the quest for super vision II. Thorofare: Slack; 2004. p.217-25.

23. Lyle WA, Jin GJ. Retreatment after initial laser in situ keratomileusis. J Cataract Refract Surg. 2000;26(5):650-9. Comment in: J Cataract Refract Surg. 2000;26(5):625-6.

24. Applegate RA, Howland HC. Magnification and visual acuity in refractive surgery. Arch Ophthalmol. 1993;111(10):1335-42.

25. Mrochen M, Kaemmerer M, Seiler T. Clinical results of wavefront-guided laser in situ keratomileusis 3 months after surgery. J Cataract Refract Surg. 2001; 27(2):201-7. 
26. Lawless MA, Hodge C, Rogers CM, Sutton GL. Laser in situ keratomileusis with Alcon CustomCornea. J Refract Surg. 2003;19(6):S691-6.

27. Urbano AP, Nosé R, Nosé W. Reoperações personalizadas. In: Alves MR, Chamon W, Nosé W, editores. Cirurgia Refrativa. Rio de Janeiro: Cultura Médica; 2003. p.359-67.

28. Febbraro JL, Buzard KA, Friedlander MH. Reoperations after myopic laser in situ keratomileusis. J Cataract Refract Surg. 2000;26(1):41-8.

29. Brahma A, McGhee CN, Craig JP, Brown AD, Weed KH, McGhee J, Brown R. Safety and predictability of laser in situ keratomileusis enhancement by flap reelevation in high myopia. J Cataract Refract Surg. 2001;27(4):593-603.
30. Zadok D, Maskaleris G, Garcia V, Shah S, Montes M, Chayet A. Outcomes of retreatment after laser in situ keratomileusis. Ophthalmology. 1999;106(12):2391-4.

31. Carones F, Vigo L, Scandola E. Wavefront-guided treatment of abnormal eyes using the LADARVision platform. J Refract Surg. 2003;19(6):S703-8.

32. Mrochen M, Kaemmerer M, Seiler T. Clinical results of wavefront-guided laser in situ keratomileusis 3 months after surgery. J Cataract Refract Surg. 2001; 27(2):201-7

33. Aizawa D, Shimizu K, Komatsu M, Ito M, Suzuki M, Ohno K, Uozato $H$. Clinical outcomes of wavefront-guided laser in situ keratomileusis: 6-month follow-up. J Cataract Refract Surg. 2003; 29(8):1507-13

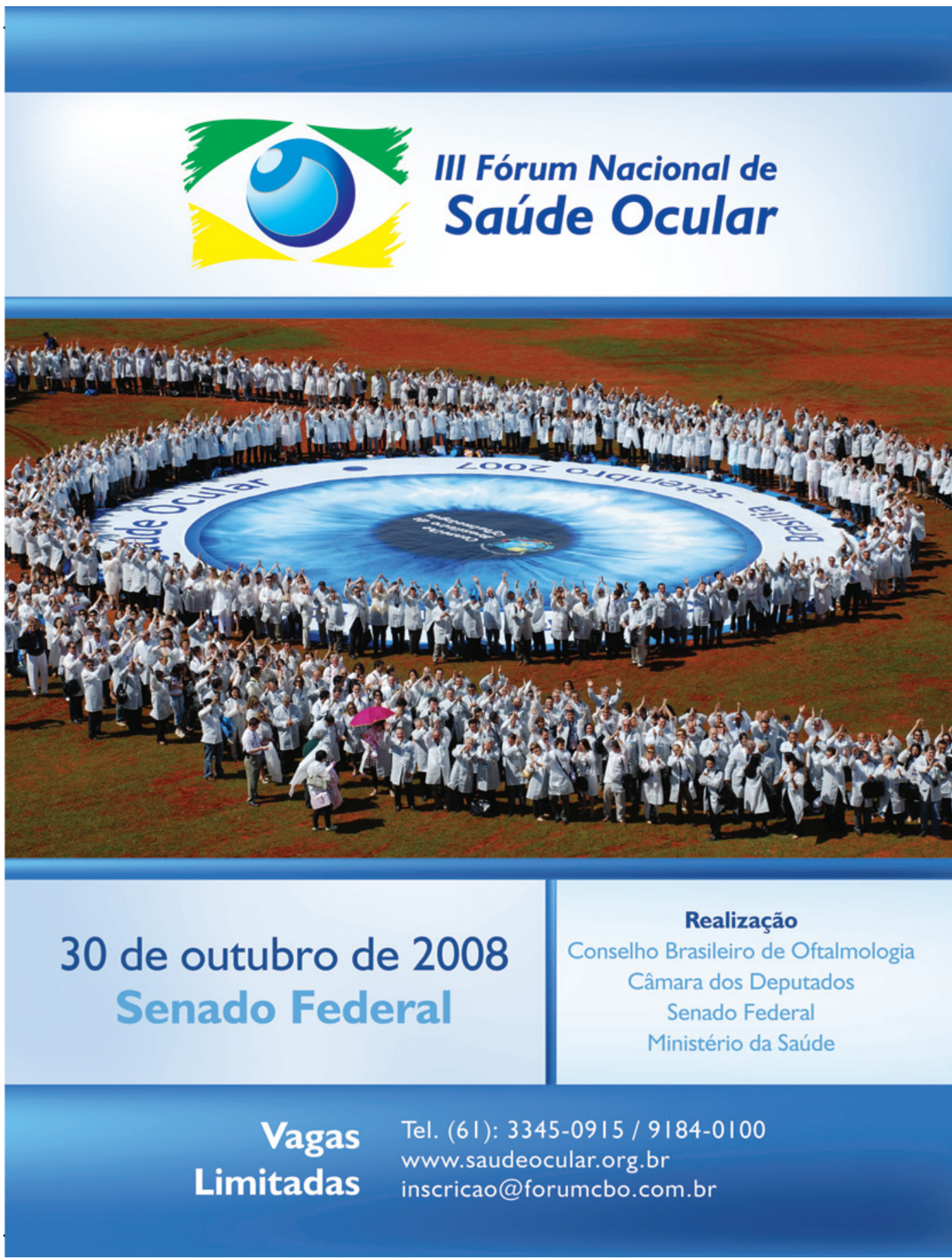

